\title{
Pharmacovigilance and Role of Packaging
}

\section{Anupam Chanda*}

Indian Institute of Packaging, Mumbai, India

*Corresponding Author: Anupam Chanda, Indian Institute of Packaging, Mumbai, India.

Received: April 24, 2019; Published: June 20, 2019

DOI: $10.31080 /$ ASPS.2019.03.0319

\section{Abstract}

Packaging plays an essential information medium through labelling and package insert(leaflet) for pharmacovigilance. Pharmacovigilance team is collecting data, identify the actual problem, assessment, monitoring and prevention of adverse effect of the Pharmaceutical products. It may happen due to wrong design of Primary Packaging device and material as well. Wrong Medication guidance like overdose and misuse and abuse of a drug as well as drug exposure during pregnancy and breastfeeding, are also of interest, even without an adverse event, because they may result in an adverse drug reaction.

Licence holder drug manufacturing company need to take immediate action once they receive and complaint from patients or from any hospitals. This information can be transmitted through SMS, Facebook, E-mail ,phone call or report to the Pharmacovigilance team.

Keywords: Pharmacovigilance; Packaging

\section{Purpose and special role}

- Before using any drugs Patients, Pharmacists, nurses and others are going through the instruction in container label and package insert those are made my experienced Packaging Technologist.

- Any special information pass to the Patient related to drug delivery need to verified by medical officer and marketing Authorization is a must.

- Selection of primary packaging material is most important to avoid product stability loss before shelf life.

- Package insert is most important since when any Physician and Nurses are not available around.

- User friendly Packaging also guarantees the good use of medicine products and promotes Patient Compliance (i.e. calendar) [1-4].

Adverse reaction of drugs can possible related to Packaging for below reasons:

1. Extractable and leachable from Glass Vial, Syringe and cartridge, rubber stopper and plunger.

2. Silica flex floats into the product due to Delamination of Glass

3. Extractable and leachable from "Polymeric Vial, syringe and cartridge.

4. Primary packaging material is not compatible with ophthalmic product.
5. Contamination of inhaler product due to "Extractable and Leachable"

6. IV Infusion contaminated due to extractables from Tube.

7. Wrong selection of Polymer in "Nitrocellulose patch.

8. Wrong design of "Autoinjector"

9. Tablet/capsule colour change in blister pack due to Extractables from Primary Packaging Material.

10. Wrong instructions in Package insert

\section{Pharmacovigilance cycle}

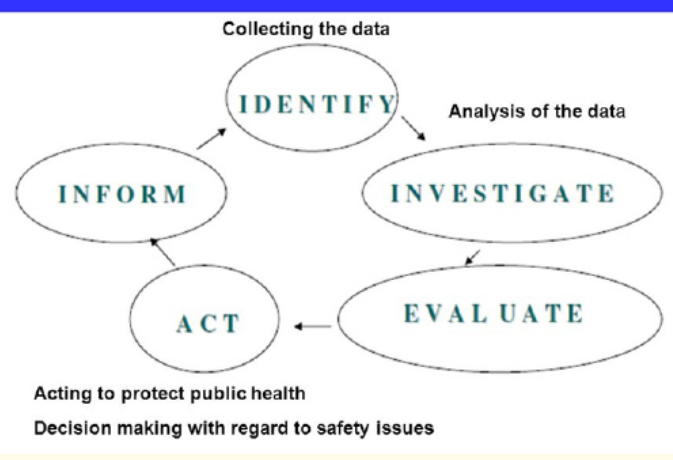

Figure 1 
Pharmacovigilance Requirements with the Registration Dossier

Pharmacovigilance System
Master File

Post Marketing Pharmacovigilance Requirements

\begin{tabular}{ccc}
$\begin{array}{c}\text { Adverse Reaction } \\
\text { Reporting }\end{array}$ & $\begin{array}{c}\text { Periodic Safety Update } \\
\text { Reports (PSUR) }\end{array}$ \\
\hline
\end{tabular}

Figure 2

Extractable and leachable from Glass Vial, Syringe and cartridge, rubber stopper and plunger.

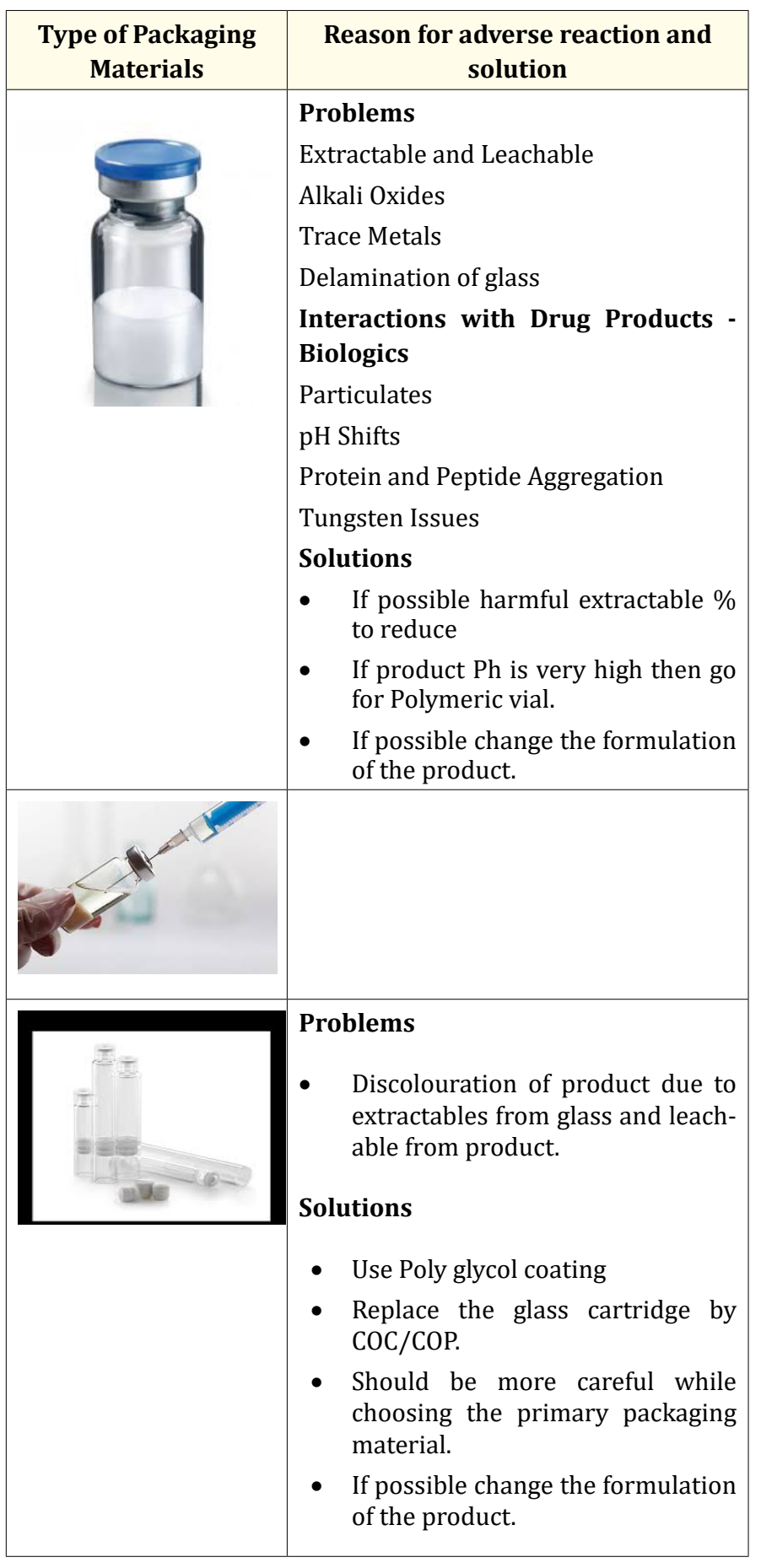

\begin{tabular}{|l|l|}
$\begin{array}{l}\text { Problems } \\
\text { Adsorption of protein from the } \\
\text { product } \\
\text { Presence of rubber particle inside } \\
\text { the product } \\
\text { Solutions } \\
\text { Coated rubber stopper to use } \\
\text { Stability need to carryout in with } \\
\text { different kind of sterilized rubber } \\
\text { stopper }\end{array}$ \\
$\begin{array}{l}\text { Problems } \\
\text { Bending of plunger rod during ap- } \\
\text { plication of drugs. } \\
\text { Colour leaching }\end{array}$ \\
$\begin{array}{l}\text { Solution } \\
\text { Check the rigidity of the plunge } \\
\text { Rod. } \\
\text { Check the Extractable of the poly- } \\
\text { meric material } \\
\text { Advisable to use "Transparent ma- } \\
\text { terial" to avoid leaching of colours } \\
\text { from plunger }\end{array}$ \\
\hline
\end{tabular}

Table 1

Silica flex floats into the product due to Delamination of Glass

\begin{tabular}{|c|c|}
\hline $\begin{array}{l}\text { Type of Packaging } \\
\text { Materials }\end{array}$ & $\begin{array}{c}\text { Reason for adverse reaction and } \\
\text { solution }\end{array}$ \\
\hline & $\begin{array}{l}\text { Problems } \\
\text { - Use of high temperature in neck and } \\
\text { along the bottom circumference of } \\
\text { the vial. As a result these area be- } \\
\text { come weak. } \\
\text { - High Ph content of the product } \\
\text { - Floating of silica flex into the prod- } \\
\text { uct. } \\
\text { Solutions } \\
\text { - Reduce the flaming temperature } \\
\text { - Iuring manufacturing of the vial. } \\
\text { the product. } \\
\text { - Use of COC/COP vial. }\end{array}$ \\
\hline & $\begin{array}{l}\text { Problems } \\
\text { - Formation metal oxide during inser- } \\
\text { tion of needle into the syringe. } \\
\text { - Delamination of inner surface of the } \\
\text { syringe. } \\
\text { Solutions } \\
\text { - If possible harmful extractable \% to } \\
\text { reduce } \\
\text { - If product Ph is very high then go for } \\
\text { Polymeric vial. } \\
\text { - If possible change the formulation of } \\
\text { the product. }\end{array}$ \\
\hline
\end{tabular}

Table 2 
Extractable and leachable from "Polymeric Vial, syringe Needle and cartridge.

\begin{tabular}{|l|l|}
\hline \multicolumn{1}{|c|}{$\begin{array}{c}\text { Type of Packaging } \\
\text { Materials }\end{array}$} & $\begin{array}{l}\text { Reason for adverse reaction and } \\
\text { solution }\end{array}$ \\
\hline $\begin{array}{l}\text { Antioxidants } \\
\text { Assure protection against thermal } \\
\text { and oxidative degradation during } \\
\text { processing and during environmen- } \\
\text { tal exposure. }\end{array}$ & $\begin{array}{l}\text { Typical Plastic additives } \\
\text { Additives, anti oxidents, stabilizers, } \\
\text { plasticezers, emulsifiers, colourants, } \\
\text { monomars, oligomers residual catal- } \\
\text { ists, impurities UV absorvers fillers, } \\
\text { anti fogging, anticrobials etc } \\
\text { Lubricants, antistatic agents, ini- } \\
\text { tiators, stabilizers, impact modifiers, } \\
\text { antioxidants, bactericides catalysts., } \\
\text { blowing agents, processing aids, } \\
\text { plasticizers, colourants, brighteners, } \\
\text { release agents, vulcanizing agents }\end{array}$ \\
\hline $\begin{array}{l}\text { Needle for Syringe } \\
\text { Problems: } \\
\text { During insertion of Needle into } \\
\text { the syringe, tungsten oxide } \\
\text { formed. } \\
\text { Sometimes corrosion observed } \\
\text { Solutions } \\
\text { Need to reduce needle insertion } \\
\text { temperature } \\
\text { Advisable to use steel needle. }\end{array}$ \\
\hline
\end{tabular}

Table 3

Primary packaging material is not compatible with ophthalmic product.

Polymers and it's standard extractable(metal) values

Acceptance criteria for E/L study in different media (one specific example)

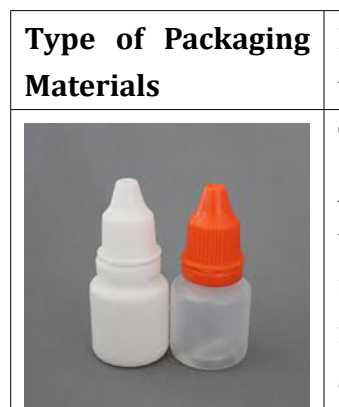

Reason for adverse reaction and solution

Typical Plastic additives:

Additives, anti-oxidants, stabilizers, plasticizers, emulsifiers, colourants, monomers, oligomers residual catalysts, impurities UV absorbers fillers, anti-fogging, anticrobials etc.

Lubricants, antistatic agents, initiators, stabilizers, impact modifiers, antioxidants, bactericides catalysts., blowing agents, processing aids, plasticizers, colourants, brighteners, release agents, vulcanizing agents

Carbonic acids:

$\mathrm{C} 1, \mathrm{C} 2, \mathrm{C} 3$

C2 - C5 -Aldehydes, Ketones, BHT derived from Irganox 1010, 1076 (BHT: 3,5-di-tert-butyl-4-hydroxytoluol), 2,5-di-tert-butyl benzene and 2,5-di-tertbutyl phenol from Irgafos 168.

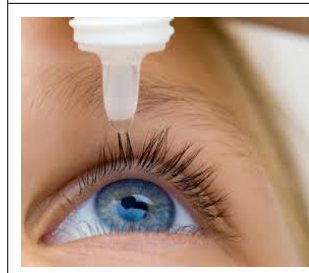

Problem

- Colour change of the product before shelf life

- Colour leaching from primary packaging materials.

- Improper dispensing of the product

\section{Solution}

- Right selection of primary packaging material is a must.

- Carryout stability studies properly.

- Carryout extractable and leachable testing of primary packaging material.

- Meter dose "Nozzle to use".

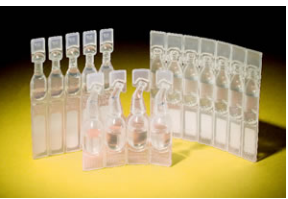

Typical Plastic additives

Additives, anti oxidents, stabilizers, plasticezers, emulsifiers, colourants, monomars, oligomers residual catalists, impurities UV absorvers fillers, anti fogging, anticrobials etc

Lubricants, antistatic agents, initiators, stabilizers, impact modifiers, antioxidants, bactericides catalysts., blowing agents, processing aids, plasticizers, colourants, brighteners, release agents, vulcanizing agents

\section{Table 4}




\begin{tabular}{|c|c|c|c|}
\hline Sl\# & Polymer & Analytics / Extract & $\begin{array}{r}\text { Component / } \\
\text { Level [ppm] }\end{array}$ \\
\hline $\mathrm{a}$ & PE & $\begin{array}{c}\text { ICPMS, ICP-OES microwave } \\
\text { digestion }\end{array}$ & $\begin{array}{c}\mathrm{Mg} / 0,5 \\
\mathrm{Si} / 16,0 \\
\mathrm{Ca} / 32 \\
\mathrm{Zn} / 1,8\end{array}$ \\
\hline $\mathrm{b}$ & LDPE & ICPMS microwave digestion & $\begin{array}{c}\mathrm{Mg} / 2,3 \\
\mathrm{Al} / 8,9 \\
\mathrm{Mn} / 0,01\end{array}$ \\
\hline c & PVC & $\begin{array}{l}\text { ICP-OES, Al / 0,2 / } \\
\text { Extraction with 5\% } \\
\text { acetic acid } 2 \mathrm{~h} 122^{\circ} \mathrm{C}\end{array}$ & $\begin{array}{l}\mathrm{Al} / 0,2 \\
\mathrm{Ca} / 0,4 \\
\mathrm{Si} / 0,9 \\
\mathrm{Zn} / 0,4 \\
\end{array}$ \\
\hline $\mathrm{d}$ & $\begin{array}{l}\text { Perfluoro- } \\
\text { elastomer }\end{array}$ & $\begin{array}{c}\text { ICP-MS, IC } \\
\text { /water } 4 \text { weeks } 80^{\circ} \mathrm{C}\end{array}$ & $\begin{array}{c}\mathrm{F} / 1,1 \\
\text { Metals }<0,1 \\
\text { TOC } 1,54\end{array}$ \\
\hline
\end{tabular}

\begin{tabular}{|c|c|c|}
\hline Compounds & Analytes & Quantification limit (ppb) \\
\hline \multirow{4}{*}{ Elements } & $\mathrm{Mg}$ & 50.0 \\
\cline { 2 - 3 } & $\mathrm{Al}$ & 10.0 \\
\cline { 2 - 3 } & $\mathrm{Cr}$ & 10.0 \\
\cline { 2 - 3 } & $\mathrm{Mn}$ & 10.0 \\
\cline { 2 - 3 } & $\mathrm{Fe}$ & 10.0 \\
\cline { 2 - 3 } & $\mathrm{Ni}$ & 10.0 \\
\cline { 2 - 3 } & $\mathrm{Cu}$ & 10.0 \\
\cline { 2 - 3 } & $\mathrm{Zn}$ & 50.0 \\
\cline { 2 - 3 } & $\mathrm{Cd}$ & 2.0 \\
\cline { 2 - 3 } & $\mathrm{Sd}$ & 2.0 \\
\cline { 2 - 3 } & $\mathrm{Pb}$ & 2.0 \\
\hline
\end{tabular}

Table 6

Table 5

\begin{tabular}{|l|c|c|}
\hline \multicolumn{1}{|c|}{ Compounds } & Analytes & Quantification limit (ppb) \\
\hline Antioxidants and UV absorbers & 2, 2- methylene- bis(4-methyl-6-tert butyl-phenol ) & 10.0 \\
\cline { 2 - 3 } & 2,6-di-tert-butyl-4-sec-butylphenol & 5.0 \\
\cline { 2 - 3 } & 2,6-di-tert-butyl-N, N- dimethylamino-p-cresol. & 10.0 \\
\cline { 2 - 3 } & 2,4-dihydroxy benzophenone. & 5.0 \\
\cline { 2 - 3 } & 2-hydroxy-4-octyloxy benzophenone & 5.0 \\
\cline { 2 - 3 } & 2-hydroxy-4-methoxy benzophenone & 5.0 \\
\hline Ethylene oxide and propylene oxide & Ethylene oxide & 0.5 \\
\cline { 2 - 3 } & Propylene oxide & 0.5 \\
\hline plasticizers & & 0.2 \\
\cline { 2 - 3 } & Butylated hydroxyl toluene & 0.2 \\
\cline { 2 - 3 } & 2- Butanone peroxide & 0.2 \\
\cline { 2 - 3 } & Di Butyl Phthalate & 0.2 \\
\cline { 2 - 3 } & 4,4- Isoprpyledene di phenol & 0.2 \\
\cline { 2 - 3 } & Benzyl Butyl Phthalate & 0.2 \\
\cline { 2 - 3 } & Di(Ethylene Glycol) Dibenzoate & 0.2 \\
\cline { 2 - 3 } & Bis(ethyl hexyl) phthalate & \\
\cline { 2 - 3 } & & \\
\hline
\end{tabular}

\section{Table 7}

Polymers are typically classified by different Criteria

\begin{tabular}{|l|l|}
\hline Origin & Natural Polymers, Synthetic Polymers \\
\hline Chemical composition & Organic Polymers, Inorganic Polymers \\
\hline $\begin{array}{l}\text { Thermo elastic } \\
\text { properties }\end{array}$ & Elastomers, Thermoplastics, Thermosets \\
\hline Route of synthesis & Chain-growth and step-growth polymers \\
\hline Number of monomers & Homo-Polymer, Co-Polymer \\
\hline
\end{tabular}

\section{Table 8}

Risk Assessment

\begin{tabular}{|l|c|c|}
\hline \multicolumn{1}{|c|}{ Solvent } & Possible Migrants & Risk \\
\hline Aqueous & Mostly Inorganics & low \\
\hline $\begin{array}{l}\text { Aqueous Buffer w/ 20\% } \\
\text { Tween 80 }\end{array}$ & $\begin{array}{c}\text { Inorganics, Siloxanes, } \\
\text { Monomers }\end{array}$ & Moderate \\
\hline $\begin{array}{l}\text { Oil Based or High } \\
\text { Organic }\end{array}$ & Monomers, Siloxanes & high \\
\hline
\end{tabular}

Table 9
Contamination of inhaler product due to "Extractable and Leachable".

\begin{tabular}{|l|l|}
\hline $\begin{array}{c}\text { Type of Packaging } \\
\text { Materials }\end{array}$ & $\begin{array}{l}\text { Reason for adverse reaction and solu- } \\
\text { tion }\end{array}$ \\
\hline & \begin{tabular}{l} 
Problem \\
Colour leaching from primary packag- \\
ing materials. \\
- Improper dispensing of the dose. \\
- $\begin{array}{l}\text { Pressing switch is not working prop- } \\
\text { erly. }\end{array}$ \\
\hline
\end{tabular} \\
\hline
\end{tabular}

Table 10 
Infusion contaminated due to extractables from Tube.

\begin{tabular}{|l|l|}
\hline $\begin{array}{c}\text { Type of Packaging } \\
\text { Materials }\end{array}$ & \multicolumn{1}{c|}{$\begin{array}{c}\text { Reason for adverse reaction and } \\
\text { solution }\end{array}$} \\
\hline $\begin{array}{l}\text { Problem } \\
\text { Colour leaching from primary } \\
\text { packaging materials. } \\
\text { Improper dispensing of the prod- } \\
\text { uct }\end{array}$ & $\begin{array}{l}\text { Solution } \\
\text { Right selection of primary pack- } \\
\text { aging material is a must. } \\
\text { Carryout stability studies prop- } \\
\text { erly. } \\
\text { Carryout extractable and leach- } \\
\text { able testing of primary packaging } \\
\text { material } \\
\text { Device validation is a must before } \\
\text { launching of the product.. }\end{array}$ \\
\hline
\end{tabular}

Table 11

Wrong selection of Polymer in "Nitrocellulose patch.

\begin{tabular}{|c|c|}
\hline $\begin{array}{c}\text { Type of Packaging } \\
\text { Materials }\end{array}$ & $\begin{array}{c}\text { Reason for adverse reaction and } \\
\text { solution }\end{array}$ \\
\hline & $\begin{array}{l}\text { Problems } \\
\text { - } \quad \text { Layer separation observed } \\
\text { - Laminate is not passed properly } \\
\text { on the skin. } \\
\text { - Colour leaching } \\
\text { Solutions } \\
\text { - Layer separation and Elongation } \\
\text { strength need to check during } \\
\text { development stage. } \\
\text { - Carryout extractable and leach- } \\
\text { able testing of primary packag- } \\
\text { ing material }\end{array}$ \\
\hline
\end{tabular}

Table 12

Wrong design of "Auto injector"

\begin{tabular}{|c|c|}
\hline $\begin{array}{c}\text { Type of Packaging } \\
\text { Materials }\end{array}$ & $\begin{array}{c}\text { Reason for adverse reaction and } \\
\text { solution }\end{array}$ \\
\hline & $\begin{array}{l}\text { Problem } \\
\text { - Improper dispensing of the product } \\
\text { - Pressing button is not working } \\
\text { properly. } \\
\text { - Improper dispensing of Dose. } \\
\text { - Needle bends } \\
\text { Solution } \\
\text { - Adequate trial need to take during } \\
\text { - } \text { development stage. } \\
\text { - Carryout stability studies properly. } \\
\text { testing of primary packaging mate- } \\
\text { - rial } \\
\text { Device validation is a must before } \\
\text { launching of the product.. }\end{array}$ \\
\hline
\end{tabular}

Table 13
Tablet/capsule colour change in blister pack due to Extractables from Primary Packaging Material.

\begin{tabular}{|l|l|}
\hline $\begin{array}{c}\text { Type of Packaging } \\
\text { Materials }\end{array}$ & \multicolumn{1}{c|}{$\begin{array}{c}\text { Reason for adverse reaction and } \\
\text { solution }\end{array}$} \\
\hline & $\begin{array}{l}\text { Reasons } \\
\text { - }\end{array}$ \\
& $\begin{array}{l}\text { Leaching from capsule shell } \\
\text { Primary packaging material is not } \\
\text { compatible with capsule shell } \\
\text { Solutions } \\
\text { Select correct Primary packaging ma- } \\
\text { terial and carryout stability study cor- } \\
\text { rectly. } \\
\text { Find out what are those extractables } \\
\text { and proceed for Alternate packaging } \\
\text { materials. }\end{array}$ \\
\hline
\end{tabular}

\section{Table 14}

Wrong instructions in Package insert

\begin{tabular}{|c|c|}
\hline $\begin{array}{c}\text { Type of Packaging } \\
\text { Materials }\end{array}$ & $\begin{array}{c}\text { Reason for adverse reaction and } \\
\text { solution }\end{array}$ \\
\hline DEFinme & $\begin{array}{l}\text { Problems } \\
\text { - Wrong dosage information printed } \\
\text { - Inadequate subject matter expertise } \\
\text { - Cut text } \\
\text { - Improper checking during proof read- } \\
\text { ing of the text matter. } \\
\text { Solutions } \\
\text { - Instructions in Packaging insert has to } \\
\text { check and approve my "Doctor" } \\
\text { - During proof regading necessary cor- } \\
\text { rection has to take if ant printing mis- } \\
\text { takes observe. }\end{array}$ \\
\hline
\end{tabular}

Table 15

\section{Bibliography}

1. Pharmacovigilance, Epidemiology and Risk Management. www.pharmalex.com

2. Pharmacovigilance and value addition for the Pharma industry, www.expressbpd.com

3. www.contractpharma.com

4. A new era of drug safety: European Pharmacovigilance, www. pharmaceutical -technology.com

Volume 3 Issue 7 July 2019

(C) All rights are reserved by Anupam Chanda. 